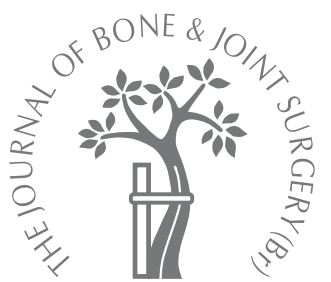

A. Terrier,

A. Reist,

F. Merlini,

A. Farron

From Ecole

Polytechnique

Fédérale de

Lausanne, Lausanne,

Switzerland

\title{
Simulated joint and muscle forces in reversed and anatomic shoulder prostheses
}

\begin{abstract}
Reversed shoulder prostheses are increasingly being used for the treatment of glenohumeral arthropathy associated with a deficient rotator cuff. These non-anatomical implants attempt to balance the joint forces by means of a semi-constrained articular surface and a medialised centre of rotation. A finite element model was used to compare a reversed prosthesis with an anatomical implant. Active abduction was simulated from $0^{\circ}$ to $150^{\circ}$ of elevation. With the anatomical prosthesis, the joint force almost reached the equivalence of body weight. The joint force was half this for the reversed prosthesis. The direction of force was much more vertically aligned for the reverse prosthesis, in the first $90^{\circ}$ of abduction. With the reversed prosthesis, abduction was possible without rotator cuff muscles and required $20 \%$ less deltoid force to achieve it.

This force analysis confirms the potential mechanical advantage of reversed prostheses when rotator cuff muscles are deficient.
\end{abstract}

In recent years the reversed shoulder prosthesis has been increasingly used in patients with glenohumeral arthropathy and partial or severe deficiency of the rotator cuff muscles. ${ }^{1-3}$ Despite the complications associated with this type of implant, it is reported as producing pain relief and greater mobility. ${ }^{4-13}$ The reverse prosthesis has two main features: it is semiconstrained, and the rotational centre of the joint is medialised. ${ }^{14}$ The constraint makes the joint more stable by balancing the deficiency of the rotator cuff muscles. The medialisation of the rotational centre increases the moment arm of the deltoid, which decreases the muscle force required to yield a given torque. This particular feature of the reverse prosthesis also compensates for the missing force of the deficient rotator cuff muscles. One problem is that the medialisation of the humerus may reduce the mobility of the arm because of impingement between the humeral component and the glenoid and between the greater tuberosity and the acromion. ${ }^{9,15}$ In addition to these kinematic limitations, failure of the humeral component has also been hypothesised because of the significant change to the muscle moment arms and the centre of rotation. ${ }^{14,16}$ Most biomechanical studies of the reversed implant do not reproduce the clinical environment appropriate for this type of implant. ${ }^{17-19}$ The aim of this study was to undertake a quantitative comparison between a reversed and an ana- tomical prosthesis. This comparison was based on the reaction force within the glenohumeral joint, the force within the deltoid and the moment arm of the deltoid during active abduction.

\section{Materials and Methods}

The reversed Aequalis (Tornier, Montbonnot, France) and the anatomical Aequalis prostheses (Tornier) were used for this comparative study. Both designs have been tested by the same finite element model of the shoulder. ${ }^{20}$ As this numerical model is already described in a previous paper, ${ }^{20}$ only the main outlines are given here. The geometry of the scapula and humerus of a normal cadaver shoulder were reconstructed from CT scans. The following muscles were included: middle deltoid, anterior deltoid, posterior deltoid, supraspinatus, subscapularis, and infraspinatus combined with teres minor. The zones of attachment of these muscles have been precisely localised. ${ }^{21}$

Active abduction was performed in the scapular plane by a synchronised contraction of each muscle. Abduction was achieved by a controlled shortening of the middle deltoid, completed by a custom-made synchronising algorithm. During abduction, the algorithm measured the force induced in the middle deltoid and then assigned a fraction of this force to the other muscles according to predefined ratios. ${ }^{20}$ As initially proposed by Poppen and 
Table I. The force amplitude of each muscle is normalised to the middle deltoid, which has a unit value by definition. Each muscle force is thus characterised by a muscle ratio, which is assumed to be proportional to its physiological cross-sectional area (PCSA) and electromyography (EMG). During abduction in the scapular plane, the EMG of the six abductor muscles are nearly proportional, ${ }^{25}$ producing constant ratios

\begin{tabular}{llll}
\hline & PCSA & EMG & Ratio \\
\hline Middle deltoid & 1.0 & 1.0 & 1.0 \\
Anterior deltoid & 1.0 & 0.8 & 0.8 \\
Posterior deltoid & 1.0 & 0.2 & 0.2 \\
Supraspinatus & 0.5 & 1.0 & 0.5 \\
Subscapularis & 1.5 & 0.3 & 0.5 \\
Infraspinatus & 1.5 & 0.3 & 0.5 \\
\hline
\end{tabular}

Reproduced with permission from Elsevier. ${ }^{20}$

Walker, ${ }^{22}$ each ratio was assumed to be proportional to the physiological cross-sectional area and electromyographic activity of the corresponding muscle. ${ }^{22-24}$ Average and constant values were estimated from the literature and normalised to the middle deltoid (Table I). ${ }^{25-28}$ Muscles were composed of a passive deformable part that could wrap around the humerus, and an active part that contained the contraction force. The passive wrapping part was represented by a ribbon-like structure around the humeral head and by a simple cable around the humeral diaphysis. The natural stabilisation of the joint was achieved by the contact force of the wrapping muscles on the humeral head and by the glenohumeral contact force. This method automatically provided the joint and muscular forces that counterbalance the weight of the arm. This algorithm was strictly validated by comparing the numerical solution to a known algebraic solution of a simplified two-dimensional model, which proved that the numerical model could solve the biomechanical system accurately. ${ }^{29}$

In the initial position, the scapula was orientated such that the scapular plane was parallel to the vertical axis and the glenoid centre line was horizontal, the diaphyseal axis of the humerus was vertical, and the articular surfaces were naturally facing. The weight of the arm was $37.5 \mathrm{~N}$, approximating to $5 \%$ of the body mass of a $75 \mathrm{~kg}$ person. It was applied at the approximate centre of gravity of the extended arm, which was $320 \mathrm{~mm}$ from the centre of the humeral head on the axis of the humeral diaphysis. ${ }^{22}$ In order to align the weight of the arm correctly relative to the muscle forces the scapula was progressively rotated according to a scapulohumeral rhythm of $2: 1 .^{30}$ Thus, when the arm was horizontal, in $90^{\circ}$ of abduction, the scapula had rotated by $30^{\circ}$ and the glenohumeral angle was $60^{\circ}$. From $0^{\circ}$ to $150^{\circ}$ of abduction there was therefore a continuous rotation of the scapula from $0^{\circ}$ to $50^{\circ}$, and a continuous increase of the glenohumeral angle from $0^{\circ}$ to $100^{\circ}$.

All components were inserted into the shoulder model according to the recommended technique (Fig. 1). For the reversed implant, the glenoid resection plane was perpen- dicular to the natural glenoid centre line, with minimal removal of subchondral bone. The base plate and the glenosphere were placed in the most inferior position to limit inferior impingement. The axis of the component was parallel to the glenoid centre line. The humeral component was also placed to preserve maximum bone support, constrained by the fitting of the stem in the medullary canal. The diameter of the glenosphere and humeral component was $36 \mathrm{~mm}$. For the anatomical prosthesis, the glenoid component axis was set coincidentally with the natural glenoid centre line. A spherical bone resection was performed with limited subchondral bone removal, and the orientation of the glenoid component was adjusted for best support on the cortical wall. The humeral component was placed to replicate the natural articular surface as closely as possible. The radius of the glenoid and humeral components were $30 \mathrm{~mm}$ and $24 \mathrm{~mm}$, respectively. With the reversed prosthesis, two extreme clinical cases were reproduced: firstly, a complete deficiency of the rotator cuff muscles, and secondly a deficiency of the supraspinatus only, preserving the subscapularis, infraspinatus and teres minor.

For both prostheses, the amplitude, direction and application point of the glenohumeral forces were calculated continuously, from $0^{\circ}$ to $150^{\circ}$ of abduction in the scapular plane. The amplitude and moment arms of the muscles were also calculated and compared. All simulations were performed with Abaqus 6.5 (Abaqus Inc., Providence, Rhode Island), in which the synchronising muscle algorithm was implemented. The stabilising contact of the wrapping muscles and the glenohumeral surfaces were solved using standard methods provided by Abaqus. ${ }^{31}$ The moment arms of the muscles were calculated with the usual tendon excursion technique. ${ }^{32}$

\section{Results}

The moment arm of each deltoid part was increased by the reversed design, particularly at the beginning of abduction (Fig. 2). In this position, the moment arm of the anterior and posterior deltoid was approximately $20 \mathrm{~mm}$ higher, but this effect decreased with greater abduction. Conversely, in the middle deltoid, the lever arm increase appeared gradually up to $110^{\circ}$ of abduction and decreased thereafter. It was also approximately $20 \mathrm{~mm}$ higher at $90^{\circ}$ of abduction. At $150^{\circ}$ of abduction the moment arms of the two implants were almost similar.

During the entire range of abduction, the joint force amplitude was reduced by $50 \%$ when the reversed prosthesis was used without any rotator cuff muscles, and by $30 \%$ when the supraspinatus only was deficient (Fig. 3). For both prostheses it increased continuously up to approximately $90^{\circ}$ of abduction (arm horizontal) and decreased thereafter. For the anatomical design the maximal force $(648 \mathrm{~N})$ was $86 \%$ of the body mass. For the reversed prosthesis, the maximal force $(313 \mathrm{~N})$ was only $42 \%$ of the body weight when all rotator cuff muscles were missing and 

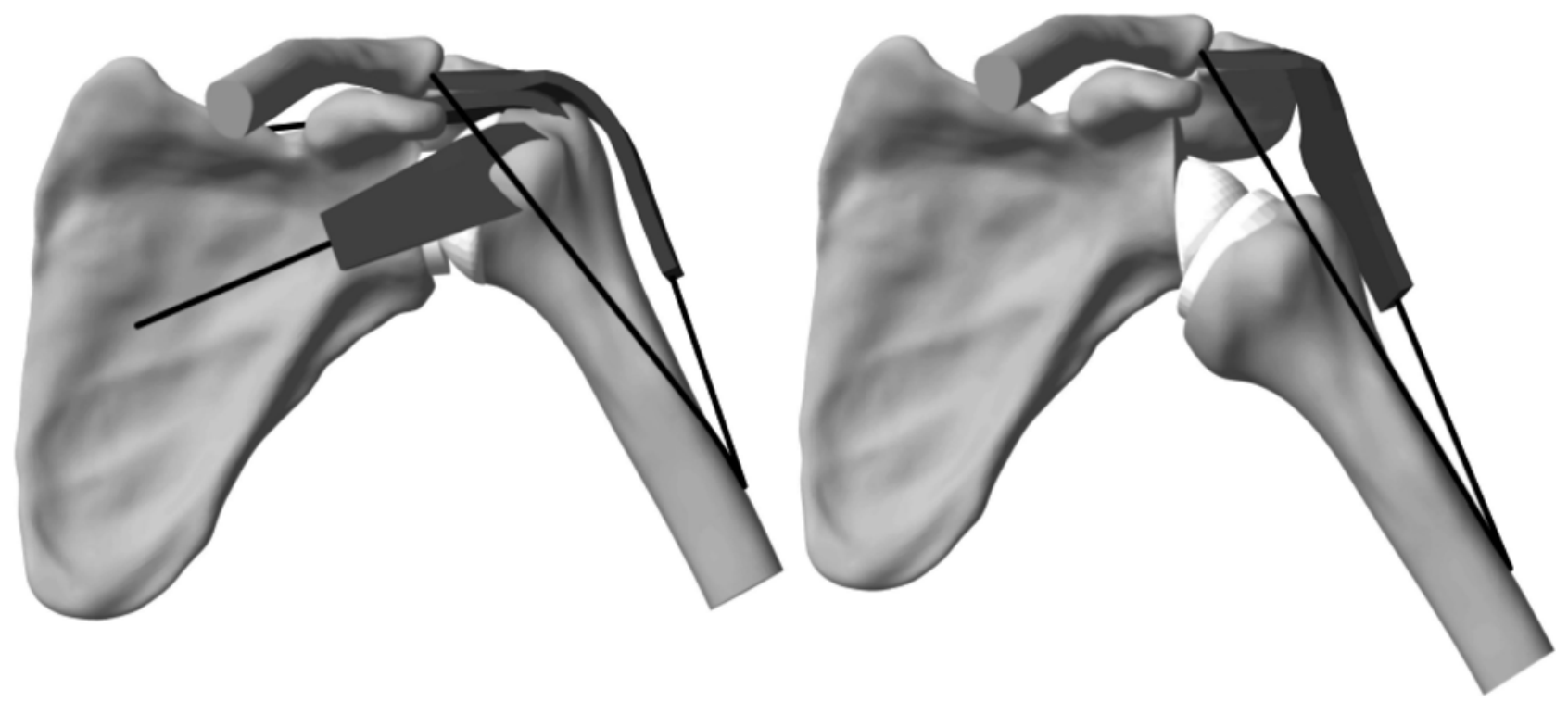

Fig. 1

Diagrams showing the location of the strip-like parts of the muscles in dark grey and the active cable-like parts in black. The reversed prosthesis (right) is shown without any rotator cuff muscle.

$62 \%(465 \mathrm{~N})$ without the supraspinatus. The direction of this force was also very different in each case (Fig. 4). For the anatomical design the contact point was initially located in the inferior border of the glenoid (rest position), but moved rapidly to the superior part during the first phase of abduction. From $30^{\circ}$ of abduction, it then moved continuously downward but remained centred. On the humeral side, the corresponding contact force moved continuously from the inferior to the superior part. For the reversed design, when all rotator cuff muscles were missing the contact point was on the superior part of the glenosphere in the rest position, but as abduction was initiated it moved immediately to its lower part. It then moved continuously from the inferior part of the centre of the glenosphere (Fig. 4). On the humeral cup, the contact point remained in approximately the same location, except at the initial resting position, where it was in the superior part. When the supraspinatus only was missing, the contact points on both surfaces were slightly more centred (Fig. 4). In all cases, the direction and location of the contact force were approximately contained within the scapular plane.

The force within the middle deltoid followed the same trend as the joint force, for both designs (Fig. 3). The maximum middle deltoid force was nearly $200 \mathrm{~N}$ for the anatomical design, and $160 \mathrm{~N}$ for the reversed case without any rotator cuff muscle.

According to the muscle ratios (Table I) and when the rotator cuff muscles were missing with the reversed prosthesis, the total maximal muscular force was $700 \mathrm{~N}$ for the anatomical prosthesis and only $320 \mathrm{~N}$ for the reversed prosthesis. The total muscle force required was thus reduced by a factor of 2 when the reversed prosthesis was used without rotator cuff muscles. When the comparison is restricted to the deltoid, this decrease was only $20 \%$. This means that $80 \%$ of the deltoid was required to perform elevation with the reversed prosthesis when all rotator cuff muscles are deficient, compared with the anatomical prosthesis. When the supraspinatus only was missing, $88 \%$ of the deltoid is required.

\section{Discussion}

This study demonstrates that the medialisation of the centre of rotation induced by the reversed prosthesis increases the moment arms of the deltoid, thereby reducing the muscle force required for abduction. With a complete rotator cuff deficiency the predicted joint force was reduced by half, but the deltoid force was reduced by only $20 \%$. When the supraspinatus only was deficient, the joint and deltoid forces were less reduced, but the contact force was more centred. In addition, the model shows that the direction and application point of the joint contact force were completely different for each design.

The shoulder model used has previously been validated against an algebraic solution and several in vitro and in vivo studies. ${ }^{20}$ In this paper, the analysis was limited to abduction in the scapular plane. This movement was chosen because of its major importance in activities of daily living, but also because of its relative simplicity. Arm elevation in this plane preserves the quasi-symmetry of the abductor muscles about that plane. This was used as an 

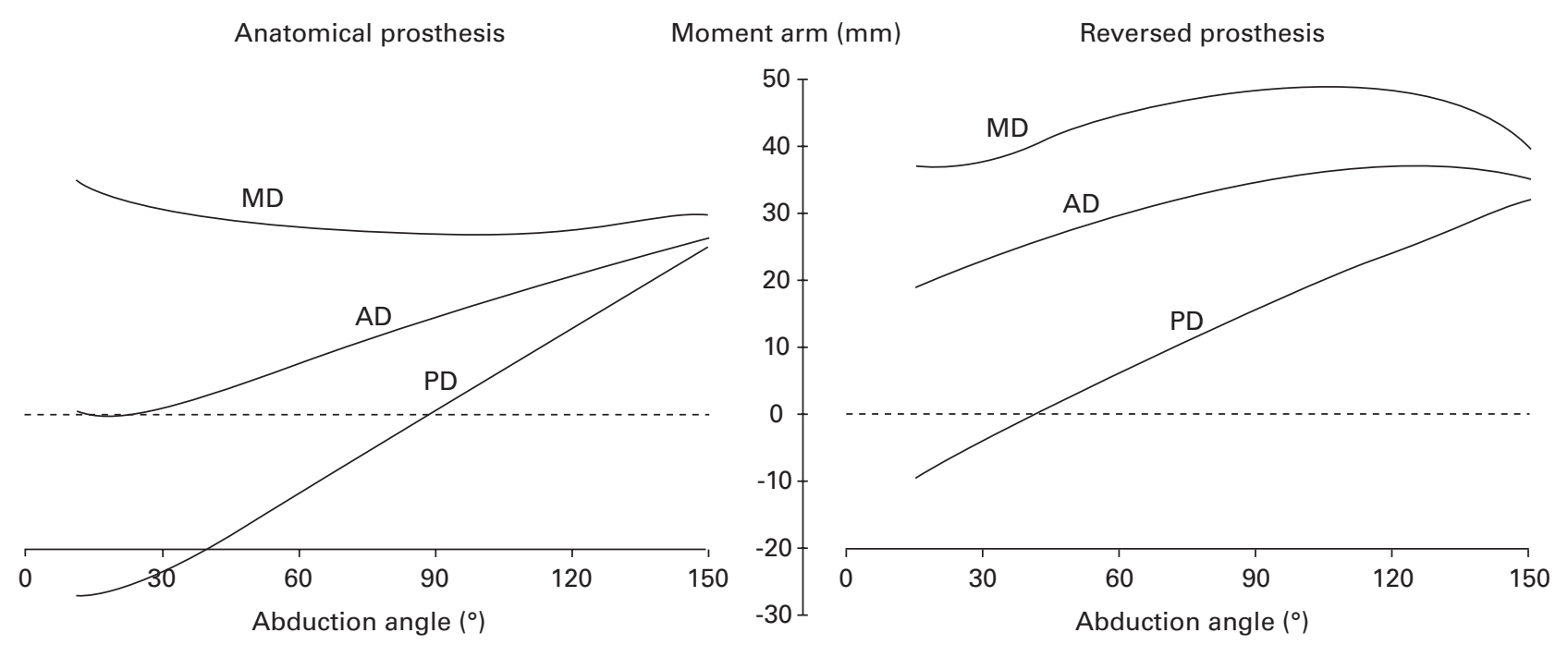

Fig. 2

Graphs showing the moment arms of the middle (MD), anterior (AD) and posterior deltoid (PD), during abduction in the scapular plane, for the anatomical and the reversed prosthesis.

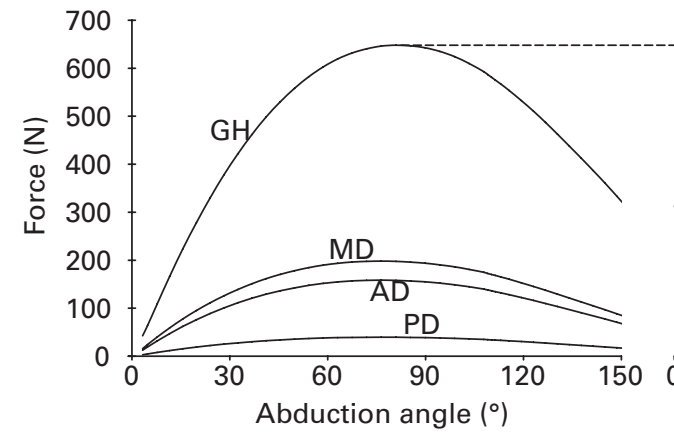
Graphs showing the forces within the glenohumeral joint $(\mathrm{GH})$, the middle (MD), anterior (AD) and posterior deltoid (PD) during abduction in the
scapular plane, for the anatomical prosthesis (left), the reversed prosthesis without any rotator cuff muscle (centre), and the reversed prosthesis without supraspinatus only (right).

argument to assume constant muscle ratios, which are roughly consistent with electromyographic measurements. ${ }^{25}$ In addition, the main advantage of the reversed design, which is the moment arm increase, is mostly effective for arm elevation, rather than for any other movement. The study was deliberately limited to a strict comparison of a reversed and an anatomical design in the same conditions. All features of the model were therefore kept identical, with the exception of the rotator cuff muscles, which were either completely or partially deactivated for the reversed implant. The muscle deactivation is of course a rough simplification of the progressive degenerative disease of the rotator cuff tendons, but as this study was limited to the consequences rather than the cause of this phenomenon, only extreme cases were considered. Because the analysis was limited to the glenohumeral joint, only scapulohumeral muscles were included in the model. Because the ligaments are known to have a slight stabilising effect for the range of movement under consideration, compared with the muscles, they were not included in the model. The long head of biceps was not considered here either, as it is usually already missing because of the initial pathology, and if not, it is tenotomised or a tenodesis is undertaken during shoulder arthroplasty. It is difficult to separate the effect of the reversed design from the muscle deficiency, as both parameters were changed in this study. We decided to assess concrete and realistic clinical cases, rather than performing a parameters analysis of a non-existent problem, such as a 


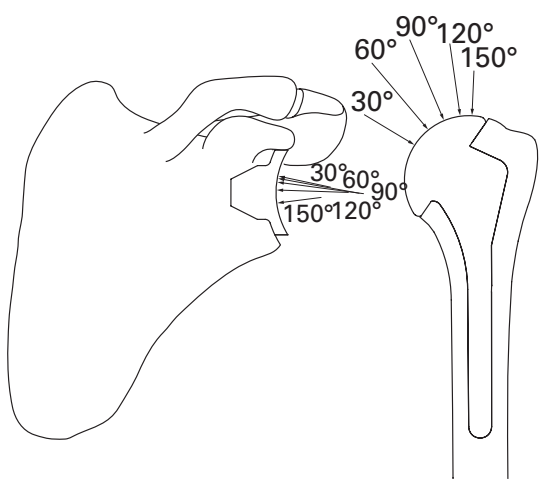

Fig. 4a

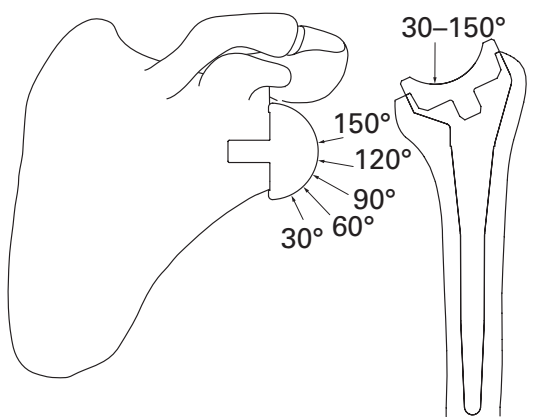

Fig. $4 b$

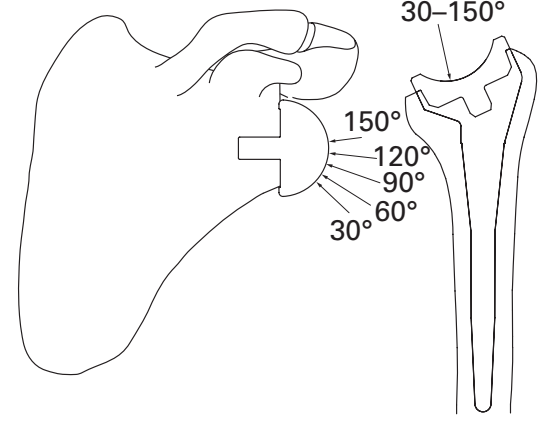

Fig. 4c

Diagrams showing the direction of the glenohumeral joint force, on the glenoid and humeral side, every $30^{\circ}$ of abduction in the scapular plane, for a) the anatomical prosthesis, b) the reversed prosthesis without any rotator cuff muscle, and c) the reversed prosthesis without supraspinatus only.

reversed prosthesis with a rotator cuff, or an anatomical prosthesis without a rotator cuff. When the arm is horizontal, the arm weight moment of force is maximal, and is twothirds balanced by the deltoid and one-third balanced by the rotator cuff muscles, according to the muscle force ratios and moment arms (Table I and Fig. 2). A complete removal of the rotator cuff muscles would increase the deltoid force by $50 \%$. On the other hand, the rotation centre medialisation of the reversed prosthesis very approximately doubles the deltoid moment arms, and would thus reduce by half the force required to balance the weight of the arm.

For the anatomical prosthesis, the amplitude and direction of the joint force were consistent with most biomechanical models of a healthy shoulder. The joint force was maximal when the arm was approximately horizontal and this has been reported previously. ${ }^{22,33,34}$ The maximum joint force was nearly equal to the body weight and this has been shown in vivo with an instrumented shoulder implant. ${ }^{35}$ The displacement of the contact point on the glenoid surface, related to the well-known rocking-horse effect, was also consistent with other models. ${ }^{22,33}$ On the humeral side, the location of the contact point also confirmed the results of a cadaver study. ${ }^{36}$

In spite of their increasing clinical use, quantitative biomechanical analyses of reversed shoulder prostheses are still rare. Another numerical model of the shoulder also reported that abduction is possible without rotator cuff muscles. ${ }^{37}$ An increase of the maximum moment arm of the deltoid was also predicted (from $35 \mathrm{~mm}$ to $52 \mathrm{~mm}$ ), inducing a reduction of the total muscle by a factor of 5 . The increase of the moment arm is consistent with our results, but the comparison of the total muscle force is not possible as that model also included the scapulothoracic muscles. In another comparative study, a shoulder model was used to calculate the moment arm of the deltoid during abduction in the scapular plane. ${ }^{38}$ The moment arms were then used to estimate the maximum muscle performance.
Although this model is only geometrical and does not solve the equations associated with equilibrium, it also reports that reversed implants increase the moment arm and the performance of the deltoid.

Although a direct comparison with clinical results is difficult, the model's predictions were consistent with clinical experience. The mechanism of the reversed prosthesis was clearly reproduced and quantified here, confirming the efficancy of this design to balance the missing stabilising and motor function of the rotator cuff muscles. The model also confirms the crucial role of the deltoid for correct function of this implant, and even predicts that activity of this muscle is reduced by $20 \%$.

The current model also provides several valuable predictions. First, the deltoid moment arms indicate that the increase in deltoid efficiency is effective mainly at the start of abduction, which means that the reversed prosthesis in particular improves the initiation of the movement. The moment arm also stresses the importance of the anterior deltoid, which can be damaged during surgery, particularly revision surgery. Concerning the joint force, the predicted reduction associated with more congruent articular surfaces should also significantly reduce the pressure on, and hence the wear of, the polyethylene component. This potential advantage could, however, be overwhelmed by the impingement between the humeral polyethylene component and the glenoid neck, which is still a major source of wear. $^{6}$ It has been assumed that the rocking-horse effect, which is neutralised at the glenoid site, might be transferred to the humeral component, weakening its fixation. ${ }^{16}$ As no rocking-horse effect was observed on the humeral component, its higher rate of failure may be associated with nonbiomechanical factors, such as a poor bone support or osteolysis.

In conclusion, this force analysis elucidates the ability of the reversed implant to allow abduction without rotator cuff muscles. The quantified gain in the moment arms was 
particularly efficient for the initiation of abduction. The specificity of the joint force in reversed prostheses confirms that this specific loading should be considered in future stress analyses, which is the next step to better estimate the long-term survival rate of these prostheses.

This study was partly supported by Tornier SAS, Montbonnot, France.

Although none of the authors has received or will receive benefits for personal or professional use from a commercial party related directly or indirectly to the subject of this article, benefits have been or will be received but will be directed solely to a research fund, foundation, educational institution, or other nonprofit organisation with which one or more of the authors are associated.

\section{References}

1. Sirveaux F, Favard L, Oudet D, et al. Grammont inverted total shoulder arthroplasty in the treatment of glenohumeral osteoarthritis with massive rupture of the cuff: results of a multicentre study of 80 shoulders. J Bone Joint Surg [Br] 2004;86-B:38895.

2. Grammont PM, Baulot E. Delta shoulder prosthesis for rotator cuff rupture. Orthopedics 1993;16:65-8.

3. Wretenberg PF, Wallensten R. The Kessel total shoulder arthroplasty: a 13- to 16 year retrospective followup. Clin Orthop 1999;365:100-3.

4. Boulahia A, Edwards TB, Walch G, Baratta RV. Early results of a reverse design prosthesis in the treatment of arthritis of the shoulder in elderly patients with a large rotator cuff tear. Orthopedics 2002;25:129-33.

5. Boileau P, Watkinson D, Hatzidakis AM, Hovorka I. The Grammont reverse shoulder prosthesis: results in cuff tear arthritis, fracture sequelae, and revision arthroplasty. J Shoulder Elbow Surg 2006;15:527-40.

6. Nyffeler RW, Werner CM, Simmen BR, Gerber C. Analysis of a retrieved delta II total shoulder prosthesis. J Bone Joint Surg [Br] 2004;86-B:1187-91.

7. Woodruff MJ, Cohen AP, Bradley JG. Arthroplasty of the shoulder in rheumatoid arthritis with rotator cuff dysfunction. Int Orthop 2003;27:7-10.

8. Wirth MA, Rockwood CA Jr. Complications of total shoulder-replacement arthroplasty. J Bone Joint Surg [Am] 1996;78-A:603-16.

9. Nyffeler RW, Werner CM, Gerber C. Biomechanical relevance of glenoid component positioning in the reverse Delta III total shoulder prosthesis. J Shoulder Elbow Surg 2005;14:524-8.

10. Wall B, Nové-Josserand L, O'Connor DP, Edwards TB, Walch G. Reverse tota shoulder arthroplasty: a review of results according to etiology. J Bone Joint Surg [Am] 2007;89-A:1476-85.

11. Levy JC, Virani N, Pupello D, Frankle M. Use of the reverse shoulder prosthesis for the treatment of failed hemiarthroplasty in patients with glenohumeral arthritis and rotator cuff deficiency. J Bone Joint Surg [Br] 2007;89-B:189-95.

12. Frankle M, Levy JC, Pupello D, et al. The reverse shoulder prosthesis for glenohumeral arthritis associated with severe rotator cuff deficiency: a minimum two-yea follow-up study of sixty patients surgical technique. J Bone Joint Surg [Am] 2006;88A(Suppl 1 Pt 2):178-90.

13. Guery J, Favard L, Sirveaux F, et al. Reverse total shoulder arthroplasty: survivorship analysis of eight replacements followed for five to ten years. J Bone Joint Surg [Am] 2006;88-A:1742-7.

14. Boileau P, Watkinson DJ, Hatzidakis AM, Balg F. Grammont reverse prosthesis: design, rationale, and biomechanics. J Shoulder Elbow Surg 2005;14(Suppl S):147-61.

15. Gutiérrez S, Levy JC, Lee WE 3rd, Keller TS, Maitland ME. Center of rotation affects abduction range of motion of reverse shoulder arthroplasty. Clin Orthop 2007; $458: 78-82$
16. De Wilde L, Walch G. Humeral prosthetic failure of reversed total shoulder arthroplasty: a report of three cases. J Shoulder Elbow Surg 2006;15:260-4.

17. Harman M, Frankle M, Vasey M, Banks S. Initial glenoid component fixation in "reverse" total shoulder arthroplasty: a biomechanical evaluation. J Shoulder Elbow Surg 2005;14:162-7.

18. Ahir SP, Walker PS, Squire-Taylor CJ, Blunn GW, Bayley Jl. Analysis of glenoid fixation for a reversed anatomy fixed-fulcrum shoulder replacement. J Biomech 2004;37:1699-708

19. Gutiérrez S, Greiwe RM, Frankle MA, Siegal S, Lee WE 3rd. Biomechanical comparison of component position and hardware failure in the reverse shoulder prosthesis. J Shoulder Elbow Surg 2007;16:9-12.

20. Terrier A, Reist A, Vogel A, Farron A. Effect of supraspinatus deficiency on humerus translation and glenohumeral contact force during abduction. Clin Biomech 2007;22:645-51.

21. Büchler $\mathbf{P}$, Farron $\mathbf{A}$. Benefits of an anatomical reconstruction of the humeral head during shoulder arthroplasty: a finite element analysis. Clin Biomech 2004;19:16-23.

22. Poppen NK, Walker PS. Forces at the glenohumeral joint in abduction. Clin Orthop 1978;135:165-70.

23. Laursen B, Jensen BR, Németh G, Sjøgaard G. A model predicting individual shoulder muscle forces based on relationship between electromyographic and 3D external forces in static position. J Biomech 1998;31:731-9.

24. Pearl ML, Perry J, Torburn L, Gordon LH. An electromyographic analysis of the shoulder during cones and planes of arm motion. Clin Orthop 1992;284:116-27.

25. Ringelberg JA. EMG and force production of some human shoulder muscles during isometric abduction. J Biomech 1985;18:939-47.

26. Kronberg M, Németh G, Broström LA. Muscle activity and coordination in the normal shoulder: an electromyographic study. Clin Orthop 1990;257:76-85.

27. Johnson GR, Spalding D, Nowitzke A, Bogduk N. Modelling the muscles of the scapula morphometric and coordinate data and functional implications. J Biomech 1996;29:1039-51

28. Holzbaur KR, Murray WM, Gold GE, Delp SL. Upper limb muscle volumes in adult subjects. J Biomech 2007:40:742-9.

29. Terrier A, Vogel A, Capezzali M, Farron A. An algorithm to allow humerus translation in the indeterminate problem of shoulder abduction. Med Eng Phys 2007 (Epub).

30. Poppen NK, Walker PS. Normal and abnormal motion of the shoulder. J Bone Joint Surg [Am] 1976;58-A:195-201.

31. No authors listed. Simulia. http://www.simulia.com (date last accessed 24 April 2008)

32. Otis JC, Jiang CC, Wickiewics TL, et al. Changes in the moment arms of the rotator cuff and deltoid muscles with abduction and rotation. J Bone Joint Surg [Am] 1994;76-A:667-76.

33. van der Helm FC. Analysis of the kinematic and dynamic behavior of the shoulder mechanism. J Biomech 1994;27:527-50.

34. Inman VT, Saunders JB, Abbott LC. Observations on the function of the shoulder joint. J Bone Joint Surg 1944;26:1-30.

35. Bergmann G, Graichen F, Bender A, et al. In vivo glenohumeral contact forces: measurements in the first patient 7 months postoperatively. J Biomech 2006;40:213949.

36. Soslowsky LJ, Flatow EL, Bigliani LU, et al. Quantitation of in situ contact areas at the glenohumeral joint: a biomechanical study. J Orthop Res 1992;10:524-34.

37. Van der Helm FCT. The 'reversed' glenohumeral endoprosthesis: the role of the rotator cuff muscles for stability and strength. J Biomech 1998;31(Suppl 1):27.

38. De Wilde LF, Audenaert EA, Berghs BM. Shoulder prostheses treating cuff tear arthropathy: a comparative biomechanical study. J Orthop Res 2004;22:1222-30. 